\title{
PLAN DE SUCESIÓN BASADO EN LA GESTIÓN POR COMPETENCIAS PARA LA CONTINUIDAD DE LA EMPRESA FAMILIAR CASO: TECNILLANTAS CAMPOS EIRL
}

\author{
SUCESSION PLAN BASED COMPETENCY MANAGEMENT FOR THE \\ CONTINUITY OF FAMILY BUSINESS \\ CASO: TECNILLANTAS CAMPOS EIRL
}

\author{
Ruth Silvia Campos Palpa, Diana Paola García Inga
}

\begin{abstract}
RESUMEN
Las empresas familiares predominan a nivel mundial, debido a que poseen una participación en el mercado que abarca de $66 \%$ a un $80 \%$, no obstante, sólo las mejores, las más competitivas, llegarán a tener éxito y alcanzarán las metas, los objetivos para que se crearon, a lo que contribuirá que la gestión de los Recursos Humanos se adapte a las nuevas estructuras económicas, estratégicas y organizativas.

Por lo tanto, hay que tener en cuenta que en la vida de las empresas familiares no hay nada inmutable ni perpetuo, nada es perfecto ni permanente en el tiempo, puesto que las organizaciones modernas se han hecho más complejas y difíciles de dirigir, constituyendo la falta de información uno de los problemas con que frecuentemente se encuentran los gestores a la hora de tomar decisiones, es por consiguiente, que la creación de un Plan de Sucesión contribuirá a que puedan tener un plan que sirva como contingencia para alguna situación imprevista y que exista un conocimiento sobre el tema, además de un personal capacitado y entrenado para asumir la dirección cuando el sucedido se retire, es decir habrá una persona capaz para afrontar las nuevas circunstancias que exista en el futuro y de esta manera permitirá identificar las situaciones, anticiparse a los problemas y facilitar la toma de decisiones. Tomando en cuenta la importancia cuantitativa y cualitativa que poseen en la economía del país y la ausencia de información sobre esta investigación; se consideró atractivo abordar este tema a manera de aportar a la generación de conocimientos.
\end{abstract}

Palabras clave: Gestión de Recursos Humanos, Plan de Sucesión

\begin{abstract}
Family businesses dominate the world, because they have a stake in the market comprising $66 \%$ to $80 \%$, however, only the best, most competitive, they will succeed and reach the goals, objectives they were created, which will contribute to the management of human resources to adapt to new economic, strategic and organizational.

Therefore, we must take into account that the life of family businesses there is nothing immutable and perpetual, nothing is perfect or permanent in time, as modern organizations have become more complex and difficult to handle, forming the lack of information one of the problems frequently encountered when managers make decisions, is therefore the creation of a succession plan that can help to have a plan that serves as a contingency for any unforeseen situation and there is knowledge on the subject, and a skilled and trained to assume leadership when the withdrawal occurred, is there someone able to cope with new circumstances that exist in the future and thereby help identify situations, anticipate problems and facilitate decision-making. Taking into account the quantitative and qualitative importance they have in the economy and the lack of information on this research, it was considered attractive way to address this issue to contribute to the generation of knowledge.
\end{abstract}

Key words: Human Resources Management, Succession Planning

\section{PLANTEAMIENTO DEL PROBLEMA}

William O'Hara (2004). Siglos de éxito. Obra. Avon: Adams Media Corporation, alude: "Antes de la corporación multinacional, existió. El negocio familiar. Antes de la Revolución Industrial, existió el negocio familiar. Antes de la Ilustración y el apogeo de la Grecia Clásica, existió el negocio familiar".

Según las estimaciones más conservadoras (Gersick, 1997) entre el $65 \%$ y el $80 \%$ de las empresas de todo el mundo son propiedad de alguna familia o están dirigidas por ellas. Algunos estudios realizados en Estados Unidos muestran que en este país el $95 \%$ de las empresas son familiares, las cuales producen el $50 \%$ del PNB y dan empleo al $42 \%$ de la población activa (Gallo, 1997, pág. 13).

Hasta hace poco más de dos décadas, surge el campo del conocimiento relativo a las empresas familiares, que aún en nuestros días carece de una adecuada integración teórica y empírica, y que no cuenta con una definición unificada de su objeto de estudio. En el caso de Latinoamérica, el estudio de las empresas familiares reviste particular importancia debido a la gran influencia de la familia sobre la empresa, y a que la inmensa mayoría de las empresas están controladas por familias.

Para Friedman y Friedman (1994), el éxito de estas organizaciones y su permanencia se correlacionan directamente con su comprensión y su planeación. Para comprenderlas, es preciso generar conocimientos sobre ellas y, afortunadamente, la información disponible crece todos los días. Una de las grandes preocupaciones de las empresas familiares se relacionan con el fenómeno de la sucesión, que es problemática para la mayoría, y probablemente el reto más difícil que alguien puede enfrentar en la dirección de una organización. Por lo general, son empresas que atraviesan por una sana situación económica, que es consecuencia de tener al frente a personas con gran visión para los negocios. Son los que "ven donde los demás mirando, no ven", y perciben que deben comprender mejor la dinámica de una empresa familiar para que la empresa nunca constituya motivo para desunir a la familia.

Tabla $N^{*} 1.1$

PARTICIPACIÓN RELATIVA DE LAS EMPRESAS

FAMILIARES EN LA ECONOMIA DEL PAIS

\begin{tabular}{|c|c|}
\hline Pals & Particlpaclon relativa (\%) \\
\hline $\begin{array}{l}\text { Austrla } \\
\text { BAlgica } \\
\text { Avemania occldental } \\
\text { Finlandla } \\
\text { Frandla } \\
\text { Relino Unido } \\
\text { Palses Bajos } \\
\text { Sulza }\end{array}$ & $\begin{array}{l}83.2 \\
69.0 \\
82.0 \\
63.2 \\
60.5 \\
67.3 \\
51.7 \\
67.2\end{array}$ \\
\hline Total & 66.0 \\
\hline
\end{tabular}

Fuente: Cabrera et al., 1999, pag. 8. Elaboracion: Begoĥa Perelra Otero 
Se estima, además, que el $40 \%$ de las 500 empresas más grandes del ranking Fortune son propiedad de familias o están controladas por ellas, para tal se muestra a continuación en la tabla $\mathrm{N}^{\circ} 1.1$.

\section{METODOLOGÍAS EMPLEADAS}

\subsection{PLAN DE SUCESIÓN}

Según el diccionario VOX de la Lengua Española, la palabra sucesión significa, entre otras cosas, la entrada o continuación de una persona o cosa en lugar de otra y prosecución, continuación ordenada de personas, cosas o sucesos. $Y$ en pocas palabras eso es lo que ha de entenderse por los planes de sucesión: la continuación ordenada y planificada de una persona en lugar de otra, con iguales 0 superiores características.

No obstante, el concepto anterior se confunde, en la mayoría de los casos, con el concepto de planes de carrera y, algunos autores, suelen referirse a ambos como sinónimos, siendo esto contrario a la verdad.

M.M. Cubeiro, J.C. Fernández (2000), menciona que los modelos de competencias, utilizados como base para la estructuración de planes de sucesión, permiten a una organización determinar los comportamientos actuales críticos, necesarios para triunfar en puestos claves, así como las competencias estratégicas necesarias para el éxito futuro.

Los planes de sucesión son utilizados por organizaciones cuya madurez responde a un orden en los procesos y a la aplicación de un modelo de competencias estructurado y alienado con su planificación estratégica. El modelo de competencias debe estar implementado en todo el personal desde que ingresa a la empresa y durante toda su vida profesional dentro de la organización. Debe ser actualizado de manera constante para que permita tener una fotografía casi instantánea del perfil de competencias de su gente para evaluar a los futuros responsables de cargos de mayor envergadura por su talento y desempeño superior.

Las Etapas del Plan de Sucesión se muestran en la Figura $\mathrm{N}^{\circ} 1$ : Figura $\mathrm{N}^{\circ} 1$

\section{ETAPAS DE UN PLAN DE SUCESIÓN}

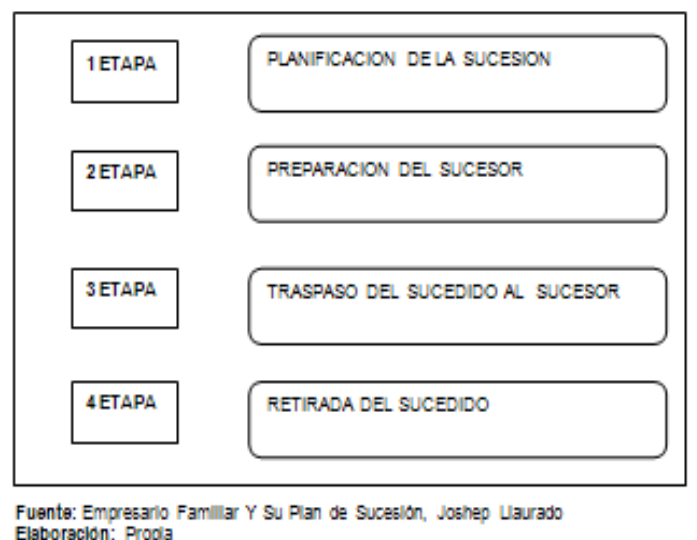

Elaboracion: Propla

\section{A. PLANIFICACIÓN DEL LA SUCESIÓN:}

En esta fase del proceso casi toda la actividad se produce fuera de la empresa y el protagonista principal es el empresario que será sucedido la tarea básica es el diseño; la familia, como propietaria, está implicada, y toma decisiones cruciales para la continuación del plan; si el plan se parase aquí, su coste sería bajo, el desgaste solo afectaría al sucesor, a su familia y, si han participado, a los colaboradores más cercanos, porque se trata de una fase previa.

Para el empresario, prevenir y planificar su propia sustitución al frente de su proyecto vital no suele ser agradable, mucho menos cuando aún se encuentra en una edad plenamente productiva; el plan es un compromiso personal, una garantía de que lo llevara a cabo, una herramienta para no caer en una simple intuición.

El Plan de Sucesión servirá para mejorar las opciones de continuidad de la empresa y para que la propiedad siga en manos familiares; es también una forma de dejar huella personal en la empresa mas allá de su presencia, una forma de controlar qué, cómo y a quién deja su legado, teniendo en cuenta que el ultimo beneficiario de un Plan de Sucesión bien hecho es su sucesor y el conjunto de la familia.

\section{B. PREPARACION DEL SUCESOR:}

Uno de los momentos claves de esta fase es la comunicación de quién es el sucesor. Se trata de un momento delicado, sobre todo si de ello depende de que se desestime a otros candidatos; asimismo mientras no se tome la decisión, todos están pendientes y la inquietud general puede distraer los objetivos más importantes. A partir del momento en que se hace la comunicación, el escogido se puede preparar mejor y sin tanta presión.

\section{TRASPASO DEL SUCEDIDO AL SUCESOR}

Incorporado y nombrado el sucesor, el relevo del sucedido era el objetivo del Plan de Sucesión; aun así, es un momento difícil, sobre todo desde el punto de vista personal. Retirarse, sin embargo, es una decisión realista que es preferible tomar uno mismo a que las circunstancias propias de la edad haga que sea una decisión forzada.

\section{RETIRADA DEL SUCEDIDO}

Como empresario una retirada a tiempo le permitirá ver la continuidad de su obra y sentirla como propia, porque suya también la responsabilidad del Plan de Sucesión; además como haya planificado su retirada, descubrirá otra formas de aprovechar su larga experiencia, ya sea en la propia empresa o fuera, en otras instituciones. Finalmente, retirarse es un signo evidente de confianza hacia su sucesor, el nuevo empresario.

\subsection{GESTIÓN POR COMPETENCIAS}

Se define al modelo de competencias, como el conjunto de conductas laborales de alto rendimiento que la organización identifica como su valor diferencial, a través del cual se desarrolla y compite exitosamente en su entorno. Muchos de los modelos desarrollados a nivel empresarial en América Latina, han partido de la escuela de McClelland, quien fue el pionero en la incorporación de competencias a la administración de recursos humanos.

Según Spencer \& Spencer (1993), una competencia es una característica subyacente de un individuo que está relacionada causalmente a un criterio de referencia de desempeño superior en un trabajo o situación.

De las aportaciones de McClelland (1973); Boyatzis (1982); Levy-Leboyer (1996) y Mertens (1992 y 1996), se deduce que en las propias características de los componentes de las competencias, se encuentran dos rasgos básicos que no se puede obviar si se quiere operacionalizar la gestión del capital humano. En primer lugar su susceptibilidad de ser observadas y medidas. En segundo lugar las posibilidades que ofrece de ser mejoradas a través de la formación o el entrenamiento.

\section{A. CARACTERÍSTICAS}

Según Spencer \& Spencer son cinco los principales tipos de competencia, a continuación se observa en la figura $\mathrm{N}^{\circ} 2$

Figura $\mathrm{N}^{\circ} 2$

MODELO DE ICEBERG

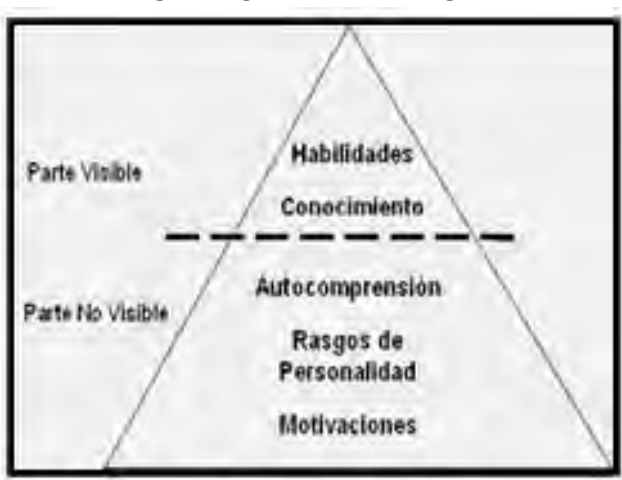

Fuente: Gesion por Competencla5. Martha Ales.

Elaboracion: Specer \& Spence 
a. Motivaciones: Lo que una persona piensa o desea y lo que la impele a la acción. Las motivaciones conducen, dirigen y seleccionan comportamientos a través de determinadas situaciones o metas.

La explicación de los términos clave de esta definición deberá ayudar a clarificar y resumir lo que los psicólogos han aprendido acerca de la motivación humana. De esta manera, básicamente un motivo puede darse cuando se piensa acerca de un objetivo con frecuencia, es decir, se trata de un interés recurrente y no de pensamientos ocasionales.

b. Características: Son las características físicas y respuestas dadas a determinadas situaciones o informaciones.

c. Auto concepto: Son las actitudes de una persona, sus valores o la imagen que tiene de ella misma.

d. Conocimientos: Los conocimientos son el tipo de competencias que más fácilmente pueden ser desarrollados o mejorados y los más fáciles de describir en su actuación, observar y medir.

e. Habilidades I Actitudes: Son susceptibles de ser mejorados con el entrenamiento, pero requieren para ser desarrollados unos rasgos personales básicos.

Estas habilidades profesionales se desarrollan básicamente con la experiencia, por ejemplo: la capacidad de negociación, el desarrollo de los colaboradores, la búsqueda de información, la presentación de datos ideas.

f. Rasgos personales básicos: Son los más difíciles de cambiar o modificar, pues su desarrollo es muy lento. Estas características, aunque relacionadas con el desempeño, son más difíciles de observar y operacionalizar en conductas y, en consecuencia, medir. Entre los rasgos personales podemos distinguir aspectos como las capacidades cognitivas, rasgos de personalidad, motivación y autoimagen.

\subsection{MODELO DE PLAN DE SUCESION BASADO EN SPENCER \& SPENCER:}

La mayoría de empresas familiares desconocen lo que un Plan de Sucesión significa, por lo que al momento de algún suceso se desconoce o no se tiene claro lo que se debe de hacer, y es por ello que en su mayoría cierran e inclusive se venden a terceros.

Es por consiguiente que se muestra un procedimiento que ayude a que las empresas tengan un Plan para el futuro (entendiendo este que puede ser a corto, mediano o largo plazo según sea el caso), para que de esta manera se tenga en consideración lo importante que significa tener un plan que ayude a que la empresa siga manteniéndose y logre sobrevivir a una siguiente generación. Para ello se presenta la figura $^{\circ} 3$, sobre el modelo de Plan de Sucesión basado en la gestión por competencias, el cual está dividido en cuatro partes las cuales son:

A. Primera Parte: En vista de que existen Empirismos Aplicativos, entendiendo esto como desconocimiento o mala práctica sobre lo que es un Plan de Sucesión en las empresas familiares, se presenta dividido en dos pasos un análisis sobre cómo se encuentra la empresa y su entorno:

- Primer Paso: Análisis de la Estructura en donde se la principal herramienta serán las encuestas para dar un informe evidente de cómo se encuentra la empresa familiar, sus colaboradores y su relación con la empresa y en que generación es en la que se encuentra actualmente.

- Segundo Paso: Planificación del Plan de Sucesión en la cual se realiza una reunión con los directivos motivo en la que se les dará una charla sobre este tema y las ventajas que tiene una empresa al contar con ello, juntamente se presentará el informe de cómo está actualmente la estructura de la empresa y sus colaboradores (Datos del primer paso), para luego a ello identificar el objetivo y alcance que tendrá el Plan.

B. Segunda Parte: Por las carencias que existe de información sobre Plan de Sucesión y la falta de un modelo que permita identificar a un posible candidato para ser el sucesor es que se realizará un programa que busque al mejor postor, mediante una serie de evidencias que se darán a conocer con instrumentos como la observación y la entrevista de tal manera que muestren datos numéricos de la situación.

C. Tercera Parte: Como anteriormente se indicó, existe carencia de información, y más aún si es que este plan se desea realizar basado en un gestión por competencias, es por ello que se realiza un diseño de perfiles previa información recopilada.

D. Cuarta Parte: Es la última parte pero la más resaltante, debido a que el tiempo y el dinero están relacionados directamente, esta parte constará de tres pasos los cuales son:

- Primer Paso: La preparación del Sucesor en donde se realizaran capacitaciones para de esta manera hacer de su conocimiento sobre lo que es el rubro, la empresa y los principales clientes y proveedores.

- Segundo Paso: El traspaso del sucedido quizás el paso donde se encuentre más emociones de todo el proceso ya que se evidenciaran muestras de alegría y nostalgia a la vez por saber que habrá alguien que manejará la empresa y por saber que no seguir aún dirigiéndolo.

- Tercer Paso: Juntamente con el segundo paso, es aquí donde se evidencia de manera más clara las emociones que el sucedido tiene.

\section{FIGURA No3 \\ MODELO DE PLAN DE SUCESIÓN BASADO EN LA GESTIÓN POR COMPETENCIAS}

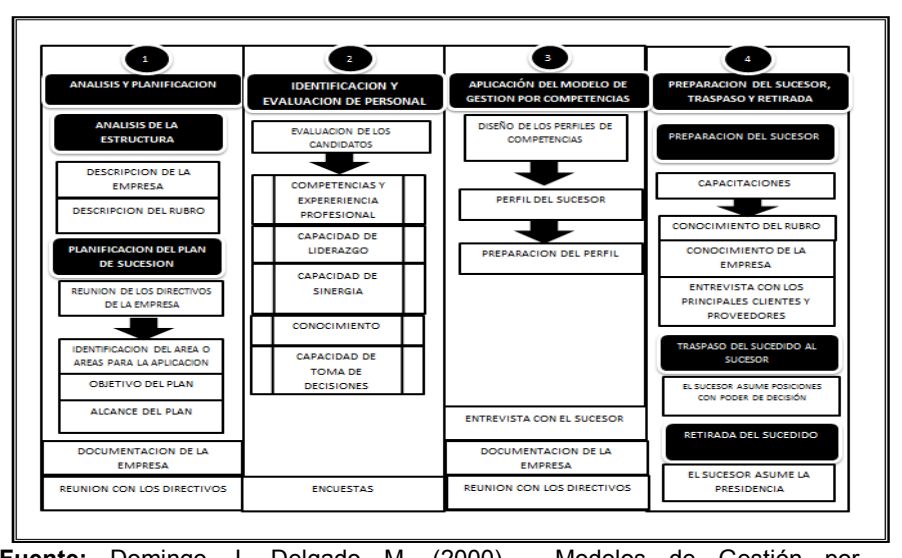

Fuente: Domingo J. Delgado M. (2000). Modelos de Gestión por Competencias. Fundación Iberoamericana del Conocimiento y EMPRESARIO FAMILIAR Y SU PLAN DE SUCESION, JOSHEP LLAURADO.

\section{DESARROLLO}

3. DESCRIPCIÓN DEL MODELO APLICATIVO 3.1. PRIMERA ETAPA: ANALISIS Y PLANIFICACION 3.1.1. ANALISIS DE LA ESTRUCTURA

A. DESCRIPCION DE LA EMPRESA: Se dará inicio con un análisis de la empresa, en la cual se evidenciará algunos puntos, mediante las encuestas de tal manera que estas puedan servir de evidencia y así poder realizar el informe final para la presentación a la Directiva de la empresa.

Asimismo se realizarán conjuntamente con la Directiva Familiar talleres para analizar la visión, misión, organigrama para de tal manera realizar algunos cambios si los hubiese con aceptación de estos.

B. DESCRIPCIÓN DEL RUBRO: Se obtendrán informes de diferentes organizaciones lineadas a sector de neumáticos correspondiente para ver la descripción del rubro, para de esta manera también aportar cual es la situación actual del rubro y cuáles son las tendencias.

\subsubsection{PLANIFICACION DEL PLAN DE SUCESIÓN}

En esta parte se realizará una reunión con los directivos familiares para de esta manera hacerles llegar el informe 
correspondiente de su actual situación en cuanto a conocimiento de Plan de Sucesión,

Luego de ello se realizará una capacitación en donde se dará a conocer el tema de importancia que es: Plan de Sucesión sus ventajas y desventajas.

Conjuntamente con la Directiva Familiar se desarrollará el objetivo que tendrá el Plan asimismo como su alcance, y el estilo de liderazgo que predomina en la empresa.

\subsection{SEGUNDA ETAPA: IDENTIFICACIÓN Y EVALUACIÓN DE PERSONAL}

\subsubsection{IDENTIFICACION DE LOS CANDIDATOS}

A. Se identifican los candidatos y su participación en la empresa.

B. Es imprescindible que al diseñar el Plan de Sucesión se debe cerciorar de que los perfiles de puesto relevantes siempre estén actualizados y alineados a la estrategia del negocio, que las competencias y habilidades requeridas se encuentran contempladas y que éstas además sean susceptibles de adecuarse conforme pasa el tiempo y las circunstancias de negocio en las que se vea inmersa la empresa. Es por ello que se tiene en cuenta en la figura $\mathrm{N}^{\circ} 4$, de los Tres Círculos en donde se analiza a cada uno de los integrantes de la empresa para poder así colocarlos en los tres lugares correspondientes los cuales son: propiedad, familia y empresa; de tal manera que se identifique a miembros de la familia, debido a que solo ellos podrán hacerse cargo de la segunda generación de la empresa.

\section{FIGURA N ${ }^{\circ} 4$}

GRÁFICO DE LOS TRES CIRCULOS

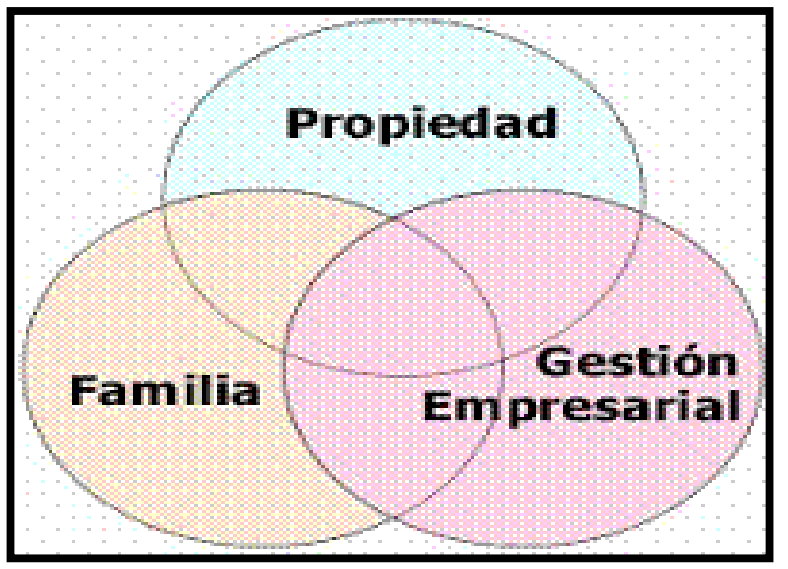

Fuente: Amat, J. 2000. La continuidad de la empresa familiar. Segunda edición. Gestión 2000.Barcelona, p. 102-103

Seguidamente se tiene el esquema que mejor explica las relaciones entre Familia, Propiedad y Empresa, el cual es el modelo de los tres círculos, formulado por Davis y Tagiuri. El sistema de la empresa familiar está formado por tres subsistemas independientes y sobrepuestos, cualquier individuo de la empresa familiar podría ser colocado en cualquiera de las áreas del gráfico.

\subsubsection{EVALUACION DE LOS CANDIDATOS}

En esta parte se tomó en cuenta las características que tiene que tener cada candidato para ocupar el puesto del sucesor de la empresa, ya que es de vital importancia, para asumir el cargo.

Una vez identificados los posibles candidatos se comenzará el proceso de selección, puesto que cada individuo posee habilidades que destacan fortalezas y áreas de oportunidad, por lo que se realizará un análisis minucioso de cada una de ellas, es primordial para seleccionar al candidato que reúna las condiciones idóneas o bien desarrollarlo en caso de ser necesario. La elección será imparcial y se fundamenta en argumentos claros y precisos, para lo cual se agrega valor al proceso proporcionando puntos de vista diferentes $e$ imparciales. Lo anterior permite reafirmar la decisión y brindar un ambiente de credibilidad y transparencia a todos los involucrados.

De esta manera se lleva a cabo el análisis por medio de tres fases de diferentes pruebas determinando los objetivos respectivos y evaluando el liderazgo, la resolución de problemas y la toma de decisiones de cada candidato; la cual es presentada a continuación en la Figura №5.

Dado el siguiente cuadro, se evidencia cada uno de las variables las cuales ayudaran a poder selección el mejor postor para el sucesor.

Por lo tanto ejecutar de manera efectiva el proceso de sucesión coadyuva a la permanencia del negocio en el largo plazo, más no lo garantiza. Es necesario enfocarse en aquellas áreas de oportunidad personales del sucesor que pudieran afectar la continuidad del negocio.

\section{FIGURA №5.}

\section{CUADRO DE EVALUACIÓN}

Elaboración: Propia

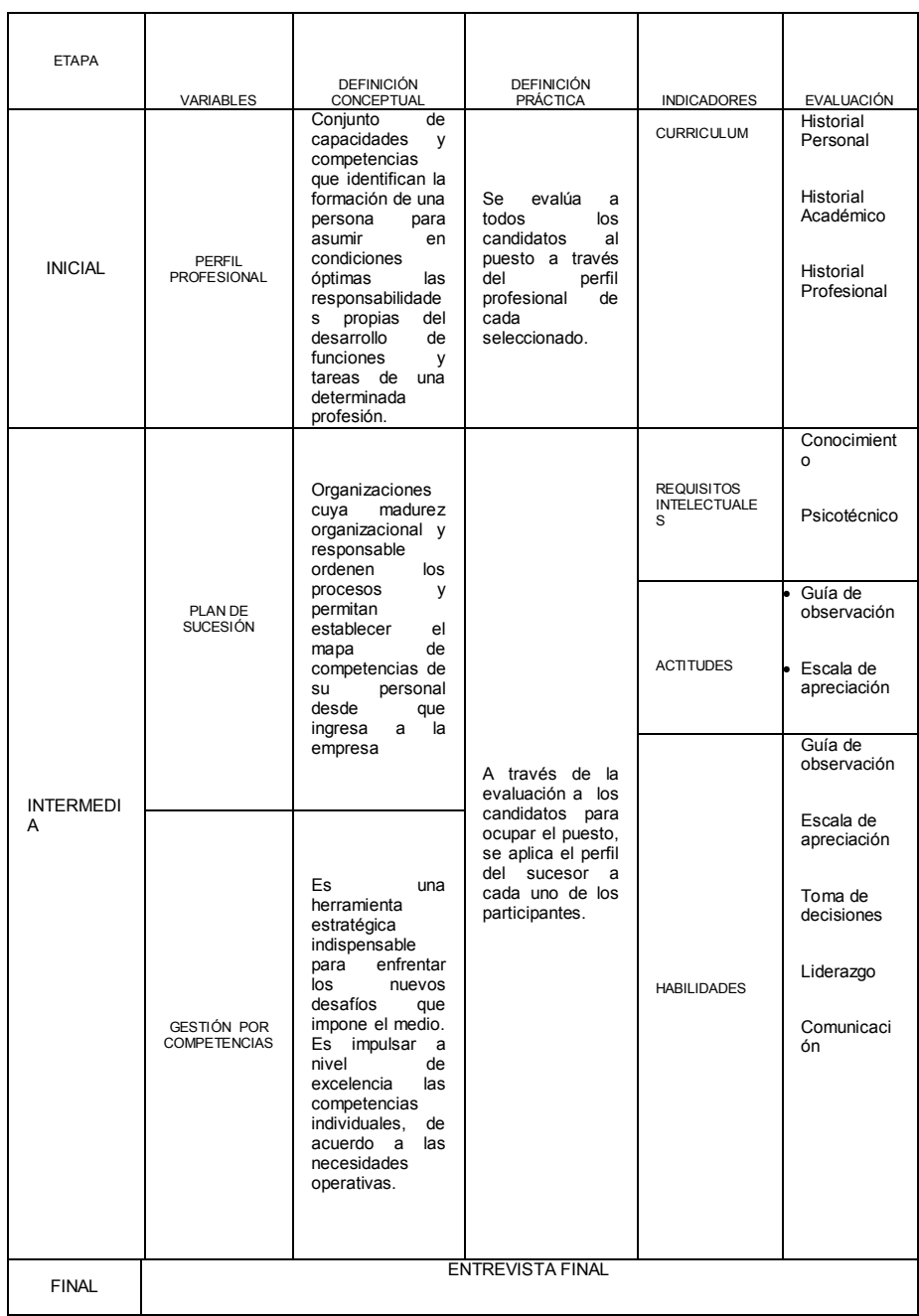

Lo que se medirá de cada uno de los candidatos será:

- Medir conocimientos: Hechos, o datos de información adquiridos por una persona a través de la experiencia o la educación, la comprensión teórica o práctica de un tema u objeto de la realidad.

- Medir competencias, aptitudes de la persona

- Habilidades cognitivas (formación académica y experiencia profesional) y habilidades interpersonales

- Definir experiencia: Se tomará mucho en cuenta, la trayectoria profesional de cada persona, grados académicos, etc.

\subsection{TERCERA ETAPA: APLICACIÓN DEL MODELO DE GESTIÓN POR COMPETENCIAS A UTILIZAR} 3.3.1. DISEÑO DE PERFIL 
En esta parte se diseña el perfil que se desea de acuerdo a la organización en donde se aplique para ello se presenta la siguiente figura $\mathrm{N}^{\circ} 6$.

FIGURA N6. DISEÑO DE PERFIL

\begin{tabular}{|l|}
\hline Diseño del perfil \\
\hline Descripción general del puesto \\
\hline Gerente General \\
\hline Principales tareas relacionadas con el puesto \\
\hline Planificar, Organizar, Controlar, Dirigir y Coordinar \\
\hline Nombre del cargo \\
\hline Gerente General \\
\hline Si es posición nueva o de reemplazo. \\
\hline Próximo sucesor de la empresa Tecnillantas Campos EIRL \\
\hline Número de personas a cargo \\
\hline 14 personas \\
\hline Posición dentro del organigrama \\
\hline Ápice Estratégico \\
\hline Cuál será su poder de decisión \\
\hline Es el que toma las decisiones de la empresa \\
\hline Responsabilidad del puesto \\
\hline Importante \\
\hline Como se va a evaluar el desempeño del puesto \\
\hline $\begin{array}{l}\text { Mediante encuestas y resultados en los Estados Financieros y } \\
\text { documentos relacionados con este. Y por la continuidad en el mercado. }\end{array}$ \\
\hline
\end{tabular}

\subsubsection{PERFIL DEL SUCESOR}

La búsqueda de potenciales sucesores es una tarea difícil debido al proceso de evaluación de los candidatos para determinar cuál de ellos cumple con el perfil necesario para la empresa y así desempeñar sus funciones de manera exitosa

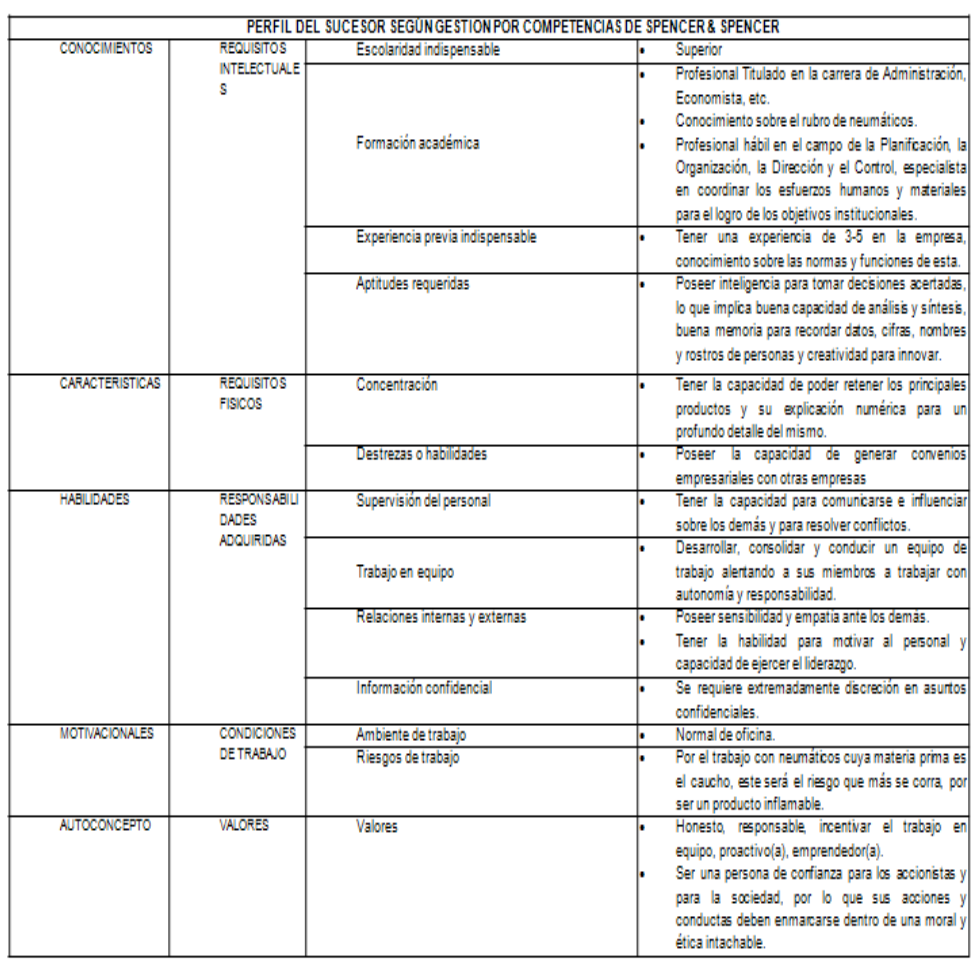

Para ello se identificará al mejor postor de acuerdo al perfil creado en la figura $\mathrm{N}^{0} 7$ según el modelo de Spencer y Spencer.

PERFIL DEL SUCESOR
Juntamente también se tendrá en cuenta las siguientes capacidades:

a. Capacidad de liderazgo

b. Capacidad de comunicación

c. Capacidad de conocimiento

d. Capacidad de toma de decisiones

\subsection{CUARTA ETAPA: PREPARACION DEL SUCESOR, TRASPASO Y RETIRADA}

3.4.1 PREPARACION DEL SUCESOR

Para ello se realizó las siguientes actividades

A. CAPACITACIONES:

Para preparar al sucesor es necesario que este tenga el conocimiento básico de:

- Historia de la empresa

- Visión y Misión

- Valores

- Instalaciones

- Ubicación geográfica

- El organigrama

- El mercado y sistemas de comercialización de la empresa

B. CONOCIMIENTO DE LA EMPRESA

Esta etapa es en la cual se brinda toda la información importante sobre la empresa, de tal manera que pueda resolver cada uno de las situaciones a las que se presente en su entorno.

\section{CONOCIMIENTO DEL RUBRO}

Si bien es cierto el rubro puede ser muy distinto y en algunas veces conocido, sin embargo existen algunos puntos esenciales que se debe tener en cuenta para algunos, es el caso de los neumáticos, puesto que cuenta con nomenclaturas diferentes en cada una de las marcas, así como sus beneficios.

\section{ENTREVISTA CON LOS PRINCIPALES CLIENTES Y PROVEEDORES}

En esta etapa tiene que hacer de su conocimiento el sucesor de sus principales clientes y proveedores de tal manera que estos conozcan al nuevo sucesor.

\subsubsection{TRASPASO}

En esta etapa la Dirección Familiar tiene la obligación de informar y reunir a cada uno de los integrantes de la Familia para poder hacer de su conocimiento el traspaso que sucederá; sin embargo de acuerdo a la organización el sucedido puede seguir en la empresa como apoyo a las gestiones del sucesor de tal manera que pueda brindar algunos alcances que le sirvan.

\subsubsection{RETIRADA}

A igual que la etapa anterior esta también es informada, sin embargo a diferencia de la anterior esta también es informada a los colaboradores, puesto que al pasar los años ya es hora de darle la oportunidad al nuevo sucesor que tome la rienda de la empresa como el desee a fin de continuar el legado que se le otorgo por sus competencias mostradas.

\section{EVALUACIÓN}

Se dará a conocer los resultados de acuerdo a una comparación entre realidades en la figura $\mathrm{N}^{\circ} 8 \mathrm{Y} 9$ quien muestra los datos proporcionados por la empresa de los cuales contrastaremos con la realidad actual de tal manera para evaluar cuál es el estado de la empresa Tecnillantas Campos con el cambio del sucesor.

Se tiene en consideración los resultados obtenidos en cuanto a la gestión del sucesor teniendo así como factores relacionados el recurso humano que es factor principal en las empresas, debido a que este es la clave de que la organización que se gestione tenga éxito; puesto que ellos son los que tienen una relación estrechamente ligada a los clientes que son la razón de ser de toda empresa. 
Responsabilidad, los propietarios pueden fortificar su negocio, reconociendo las fortalezas de su cultura familiar y transmitiéndolas a la familia y a los empleados, ya que la articulación de éstas son el punto de partida donde convergen la visión patrimonial y la visión corporativa del negocio. Cuando los valores no son claramente transmitidos, la cultura empresarial se debilita al igual que el compromiso de los propietarios, de hecho, las familias se enorgullecen cuando ven reflejados sus valores en las fortalezas de su cultura empresarial, y este es un activo intangible que consolida el patrimonio familiar.

En este sentido, la mejor forma y tal vez la más correcta de transmitir los valores familiares a la empresa, es a través del ejemplo. Así, en cabeza de los propietarios recae la responsabilidad de crear una cultura bien orientada: llegando a tiempo, con honestidad, teniendo excelentes relaciones interpersonales, haciendo negocios transparentes, demostrando cualidades para el cargo; son factores que influyen poderosamente en el fortalecimiento de los valores internos y que se ven reflejados al público.

Finalmente, está la debida articulación de los objetivos y metas financieras del negocio con la visión y los valores familiares, que es también responsabilidad de los propietarios, para la fijación de expectativas razonables en cuanto al desempeño del negocio y en este punto, los propietarios deben tener metas claras en cuatro áreas financieras del negocio: crecimiento, riesgo, rentabilidad y liquidez, de tal manera que la empresa pueda tener una continuidad en el mercado.

FIGURA N8

ANALISIS DE REALIDADES

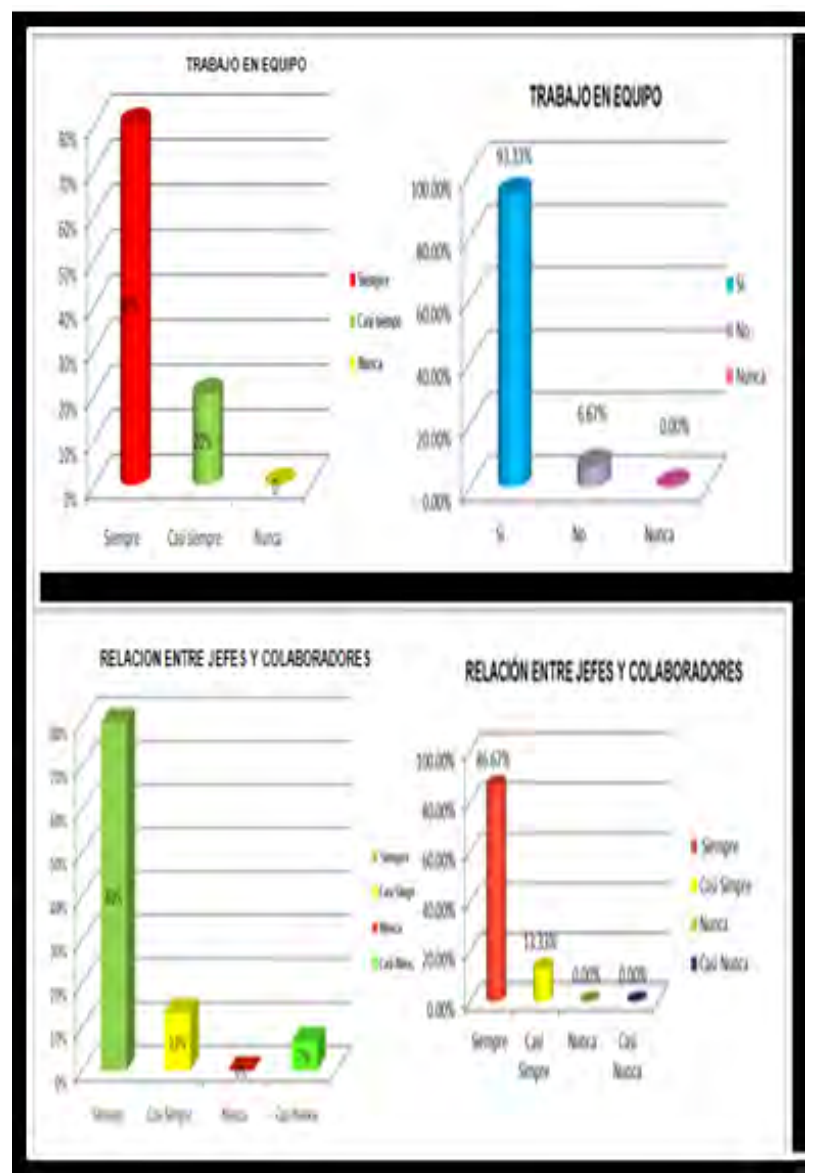

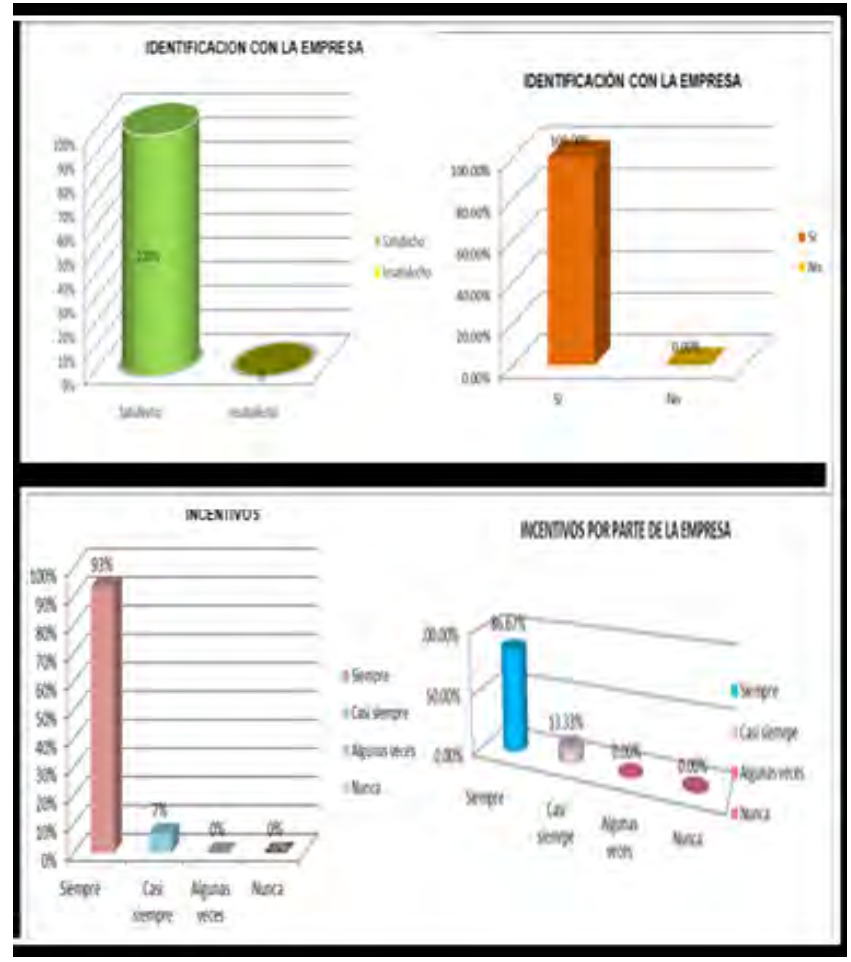

FIGURA N ${ }^{\circ} 10$

ANALISIS DE REALIDADES

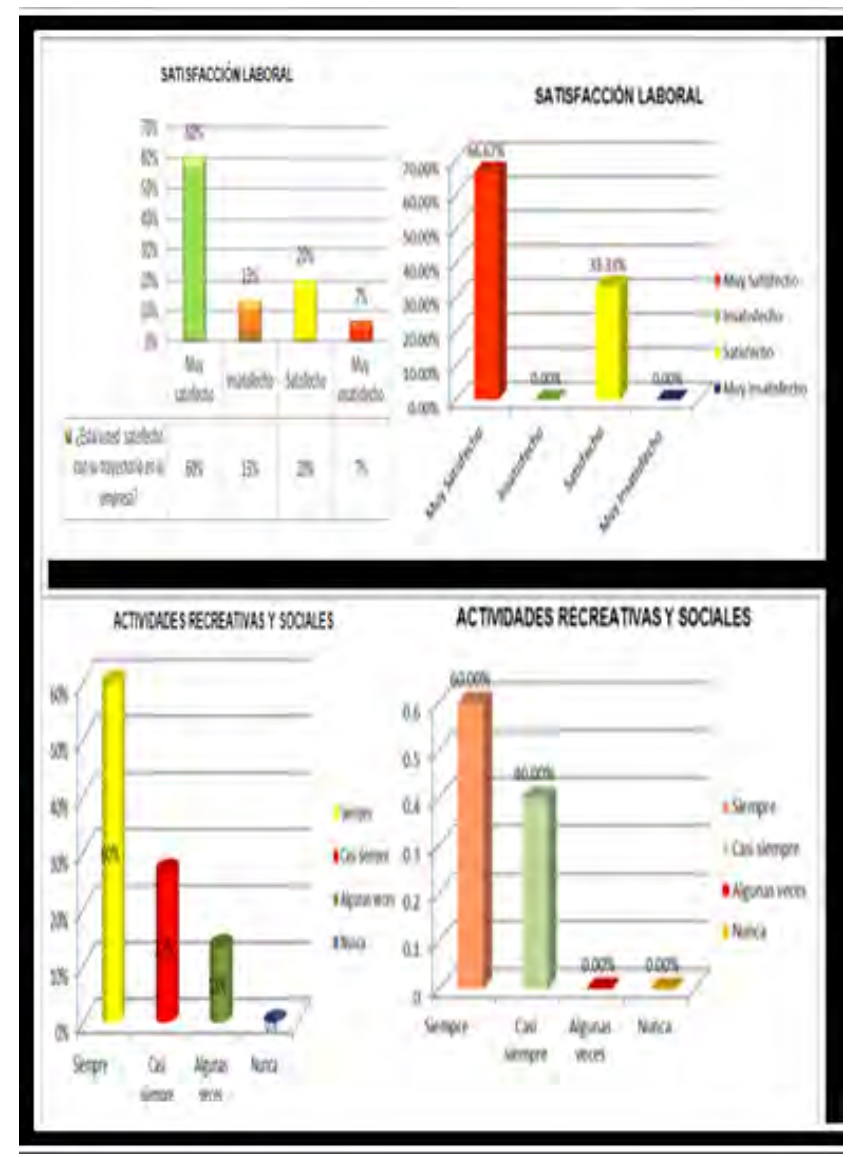




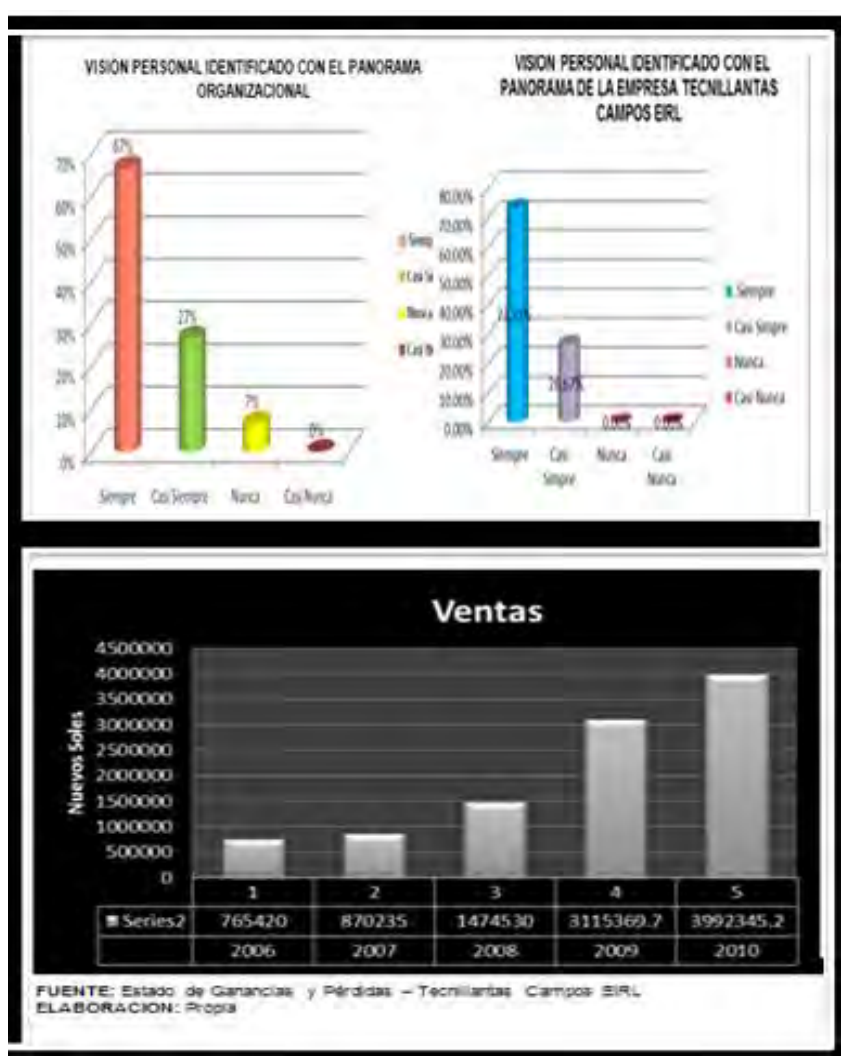

\section{CONCLUSIONES}

a. Se afirma que existen Empirismos Aplicativos, que se presentaron para la realización del Plan de Sucesión; esto debido a que tanto como en la empresa Tecnillantas Campos EIRL como en otras empresas desconocen este tema, por ende existe pocas empresas que lo ponen en práctica, ya sea por carencias de presupuesto que puede llegar a tener, o por algunas limitaciones de este tema como el perfil de un sucesor, así como también el tema familiar (en este caso podría ser las riñas que existiese entre familia por la deliberación y aceptación de un sucesor) que embarga este contexto.

b. El hecho de crear un Plan de Sucesión, ayuda a que la continuidad de la empresa Tecnillantas Campos EIRL, avance para un norte, debido a que se pudo capacitar y elegir a la persona adecuada de acuerdo a un modelo de Gestión por Competencias( Spencer \& Spencer), debido a que este autor plantea que las competencias están compuestas por características que incluyen los rasgos psicofísicos, las formas de comportamiento, el auto concepto, los conocimientos, destrezas manuales, las destrezas mentales 0 cognitivas y la motivación, este último puesto que todo individuo trabaja por un motivo en su vida ya sea sobrevivir en su trabajo para su alimento, para su familia, o para conseguir lo que desee.

c. Se evidencio la utilidad que tuvo el Marco Teórico debido a la importancia que tiene el Plan de Sucesión para una empresa, como se mostró existen porcentajes reales los cuales en su mayoría solo llegan hasta la segunda generación y muy poco porcentaje a la tercer, es por ello que este plan podrá ayudar a que sobreviva a lo largo de generaciones.

d. Un factor importante en la empresa Tecnillantas Campos EIRL y en toda organización es el talento humano, prueba de ello es que se utilizó la Gestión de Competencias, en donde cada uno de los postores pudiesen demostrar lo competentes que son para asumir el cargo de sucedido, todo ello de acuerdo a cada una de las normas y políticas de las organizaciones.

e. Actualmente las empresas desconocen de este tema importante y que puede ser de gran ayuda para estas, puesto que al tener un Plan que sirva para una contingencia (muerte o enfermedad del sucesor) o para tener una dirección de lo que sucederá en el futuro, planificara de una manera más ordenada y especifica pudiendo evitar conflictos entre familia $e$ inclusive generando de esta manera una siguiente sucesión teniendo como modelo el que ya se presentó.

f. Se muestra que la creación de un Plan de Sucesión basado en un Modelo de Gestión por Competencias, aporto que se lleve de una manera ordenada en la cual la familia y colaboradores lo lleven a cabo de una manera exitosa, probando así que este modelo ayudará a que la empresa Tecnillantas Campos EIRL, siga en el entorno competitivo que posee a lo largo de la generación que se encamina.

g. Muchas veces se cree el que implantar algo nuevo generará cambios y estos a su vez traerán gastos que la empresa no debiera de realizar, sin embargo con esta propuesta de modelo que se presentó se puede evidenciar que el crearlo juntamente con toda la familia y esta a su vez teniendo conocimiento pudiendo alcanzar algunos puntos de importancia para la empresa, debido a que la continuidad de esta dependerá en muchos casos la situación familiar de las familias que también integran por parte de los colaboradores, debido a que esta empresa en muchos de los casos es el sostén familiar, y es por ello que importará mucho que la organización crezca de tal manera que siga manteniendo satisfechos a los clientes, pero esto se llevará a cabo gracias a los colaboradores que si se sienten satisfechos también brindarán un buen servicio a estos, de tal forma que todos puedan ganar, y asimismo la generación de la empresa también pueda continuar.

\section{VII.- RECOMENDACIONES}

h. Se sugiere poder hacer de su conocimiento a cada uno de los interesados, teniendo como base la presente investigación en donde se dio a conocer diversos puntos, pudiendo resolver algunas inquietudes que las empresas poseen de tal manera que evitemos poder caer en desconocimiento del tema o aplicar mal en otras organizaciones.

i. Al momento de capacitar al personal tener en cuenta que el modelo de gestión por competencias que se utilizó no es la única herramienta, sin embargo, puede servir de gran ayuda para los que les interesa llevar a cabo como materia de estudio especifica. E inclusive para otros pueden utilizarlo como base para materia de antecedente de estudio sobre el modelo aplicado.

j. Por otra parte se recomienda que la empresa Tecnillantas Campos EIRL, siga con actualizándose sobre este tema, y por el contrario que no sea una más que se quede solo en la segunda generación sino por el contrario que exista una continuidad en el mercado sobre esta empresa.

k. Se sugiere que se continúe con una adecuada gestión competente, puesto que ello conllevará a que se sigan realizando las capacitaciones que actualmente se da en la empresa, debido a por ser una empresa perteneciente al rubro automotriz, necesita estar actualizándose velozmente por las variantes que existen en el caso específico de neumáticos (variedad de modelos, números y marcas que se comercializa actualmente en el mercado). 
I. Se recomienda no dejar de lado este plan una vez terminado el proceso de segunda generación, mas por el contrario se sugiere llevar una reunión con cada uno de los directivos familiares a fin de que se pueda crear un protocolo familiar conciso llevando consigo puntos diferentes de cada uno de los que tendría parte en la empresa.

m. Se recomienda hacer de su conocimiento a la familia, en todo momento de decisión puesto que esto se verá afectado en la dirección del nuevo sucesor, pudiendo así evitar los conflictos futuros que se presentase.

\section{VIII.- REFERENCIAS}

\subsection{REFERENCIAS BIBLIOGRÁFICAS}

a. Caballero Romero, Alejandro. Guías Metodológicas para los planes de tesis de Maestría y Doctorado. Primera Edición. Lima: Instituto Metodológico Alen Caro 2009,450 Páginas

b. Finch Stoner, James Arthur, Freeman Edward, R. Gilbert Daniel. Administración. Sexta Edición. Publicado por Pearson Educación. Impresa en México 2007,456 páginas

c. Idalberto Chiavenato. Administración de Recursos Humanos. Quinta Edición. McGRAW-HILL INTERAMERICANAS.A.

d. Robert N. Lussier y Cristopher F.Achua. Teoría, Aplicación de habilidades. Segunda Edición. Thomson 